\title{
Creeping solitons in dissipative systems and their bifurcations
}

\author{
Wonkeun Chang, Adrian Ankiewicz, and Nail Akhmediev \\ Optical Sciences Group, Research School of Physical Sciences and Engineering, The Australian National University, \\ Canberra, ACT 0200, Australia \\ J. M. Soto-Crespo \\ Instituto de Óptica, CSIC, Serrano 121, 28006 Madrid, Spain
}

(Received 13 April 2007; published 26 July 2007)

\begin{abstract}
We present a detailed numerical study of creeping solitons in dissipative systems. A bifurcation diagram has been constructed for the region of transition between solitons and fronts. It shows a rich variety of transitions between various types of localized solutions. For the first time, we have found a sequence of period-doubling bifurcations of creeping solitons, and also a symmetry-breaking instability of creeping solitons. Creeping solitons may involve many frequencies in their dynamics, and this can result, in particular, in a multiplicity of zig-zag motions.
\end{abstract}

DOI: 10.1103/PhysRevE.76.016607

PACS number(s): $05.45 . \mathrm{Yv}, 42.65 . \mathrm{Tg}, 47.20 . \mathrm{Ky}$

\section{INTRODUCTION}

In general, nonlinear dissipative systems possess stationary and moving localized solutions. For a given set of equation parameters, the form of the solution is usually set, so only one exists for a specified situation. Occasionally there can be a few, but we do not have a family of them. Dissipative systems also have pulsating solutions, i.e., solutions that oscillate periodically along the propagation direction. These are limit cycles in an infinite-dimensional phase space and, as such, they are also fixed, i.e., the period, shape dynamics, and velocity as a function of propagation distance are also fixed. Changing the parameters of the system allows us to change the characteristics of the solution. As in finitedimensional nonlinear systems, the soliton parameters can change continuously as well as via bifurcations. In particular, the velocity of solitons and pulsating solitons can change abruptly at the point of the bifurcation.

The complex Ginzburg-Landau equation (CGLE) is a generic equation that models a variety of phenomena in systems far from equilibrium [1]. Examples include pulse generation in passively mode-locked lasers [2], superconductivity, superfluidity, Bose-Einstein condensation, and strings in field theory. The variety of these systems is enormous. Moreover, the number of solutions to this equation, even in the case of a cubic nonlinearity, is so large that the sphere of this knowledge by itself has been called "the world of the Ginzburg-Landau equation" [1]. Even if we restrict ourselves to the localized solutions of the CGLE, we are faced with highly complicated regions of soliton existence and intricate bifurcation diagrams. Our present work is an attempt to widen the knowledge of soliton transformations and, in particular, of solitons that move transversely while they are pulsating. To be specific, we shall present the results of numerical simulations that show bifurcations from stationary to pulsating solitons and, further, from pulsating to creeping ones, i.e., when the velocity of the soliton changes from being zero to a non-zero function of the propagation distance. Solitons in dissipative systems can, indeed, have very complicated bifurcation diagrams. The examples that we present here demonstrate this complexity.
It has been shown in previous studies [3,4] that there is a specific range of parameter values where solitons in dissipative systems are transformed into fronts. Consequently, other research showed that this is not a simple transformation [5]. In sequence, it splits into a transformation from a plain soliton into a pulsating one and a composite pulse as intermediate steps. Our present research further enriches our knowledge about this region. It turns out to be filled with a rich variety of bifurcations between stationary, pulsating, and creeping solitons. In particular, the region of parameters where creeping solitons exist happens to have an elaborate collection of various types of soliton solutions. They may appear as separate branches of solitons, or they may bifurcate from another family of solutions. A careful study of these solutions helps us to understand the general dynamics of solitons in dissipative systems.

We have found period-doubling bifurcations of creeping solitons and a further transformation into chaotic moving solitons through a sequence of period-doubling bifurcations. We have uncovered a symmetry-breaking bifurcation from a plain pulsating soliton into a creeping soliton. We have also found two different families of creeping solitons with zig-zag motion that may exist simultaneously. We have also determined that creeping solitons can exist for the same set of parameters as stationary solitons with zero and non-zero velocities. Simple modeling of all these phenomena using the method of moments [6,7] does not seem possible at this stage. The method of moments is a "broad brush" approach and is best applied to solitons which are well-approximated by simple functions which do not require many solution parameters. At any rate, it would be difficult to apply when using one trial function for all the types of solitons described here. On the other hand, some solutions have such a complicated behavior that it is doubtful that there would be any trial function that would allow a reduction to a simple lowdimensional dynamical system. Thus, in this work, we restrict ourselves to presenting our numerical discoveries.

\section{MODEL}

The cubic-quintic CGLE, in dimensionless form, can be written as [5] 


$$
\begin{aligned}
i \psi_{t} & +\frac{D}{2} \psi_{x x}+|\psi|^{2} \psi+\nu|\psi|^{4} \psi \\
& =i \delta \psi+i \epsilon|\psi|^{2} \psi+i \beta \psi_{x x}+i \mu|\psi|^{4} \psi,
\end{aligned}
$$

where $t$ and $x$ are the propagation and spatial variables, respectively, and $\psi(x, t)$ is the normalized envelope of the field. $D$ is the dispersion coefficient, with the $D= \pm 1$, depending on whether the dispersion is anomalous or normal, respectively. $\delta$ is the linear gain-loss coefficient, $\beta$ accounts for spectral filtering, $\epsilon$ represents the nonlinear gain, $\mu$ characterizes the saturation of the nonlinear gain, and $\nu$ is the coefficient of the quintic nonlinearity. During the numerical computation of the propagation equation, the magnitude that we most often monitor is the energy, $Q$, carried by a certain solution after a propagation distance $t$. It is defined by

$$
Q=\int_{-\infty}^{\infty}|\psi(x, t)|^{2} d x .
$$

When $Q$ oscillates on propagation, we denote its maxima and minima by $Q_{\max }$ and $Q_{\min }$, respectively.

\section{NUMERICAL SIMULATIONS}

The CGLE admits a variety of localized pulsating solutions, as well as period-doubling bifurcations [8]. In order to investigate soliton bifurcations in a finite region bounded by a set of parameters, we have solved Eq. (1) using a split-step Fourier method.

In most cases, the simulations were carried out using a numerical grid of 2048 points in $x$, and with a step size $\Delta t$ $=0.005$. It is important that the results obtained are the true approximated solutions of the CGLE and not a consequence of numerical artifacts, so the solutions have been verified using finer resolutions in both $x$ and $t$ directions. All the results presented in the paper passed a final verification with 16384 points in the $x$ direction and with $\Delta t=0.0001$. Most of the results presented here have been verified by running two independently created codes on different computers.

A set of parameters that is known to give a creeping soliton with double period is the following: $D=+1, \epsilon=0.835$, $\delta=-0.1, \beta=0.08, \nu=-0.08$, and $\mu=-0.11$. We first solved Eq. (1) for this set of parameters with an asymmetric initial condition. Once it converges to the creeping soliton (see Ref. [8]), we use this solution as the initial condition for finding a new solution for a different set of equation parameters in the vicinity of the first one, viz. we change $\epsilon$ slightly while keeping all the other parameters fixed. By repeating this procedure, we were able to find out how the solution changes its behavior as $\epsilon$ is changed. More specifically, starting from the above set of parameters, $\epsilon$ has been varied in both directions, ranging up to 0.85 and down to 0.78 with a step $\Delta \epsilon$ $=0.0002$. When an abrupt change occurs due to the jump of the solution from one type to another, we carefully study the vicinity by changing $\epsilon$ in both directions with a smaller increment. In this way, we uncover some hysteresis cycles and their corresponding co-existing different solutions. For every value of $\epsilon$, we take the maxima and minima values of the soliton energy, $Q$, along the propagation direction, once any

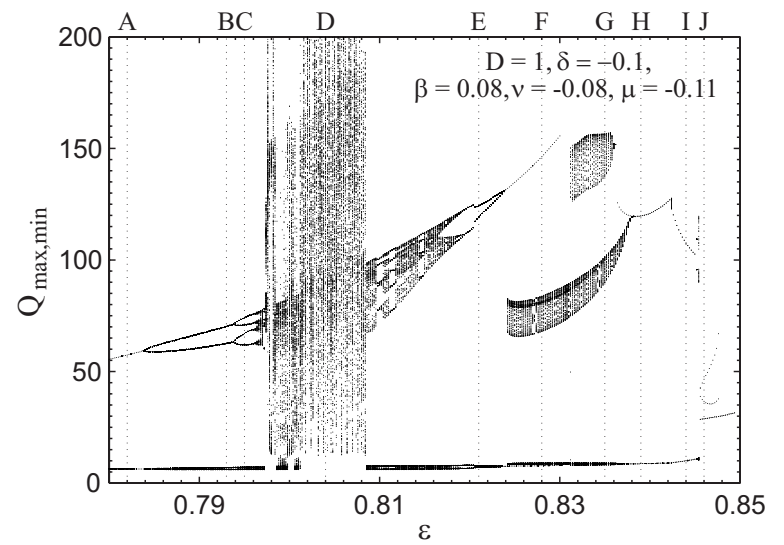

FIG. 1. Soliton energy bifurcation diagram for varying $\epsilon$. The rest of parameters are fixed: $D=1, \delta=-0.1, \beta=0.08, \nu=-0.08$, and $\mu=-0.11$. The values of $\epsilon$ marked with vertical dashed lines and labeled $A$ to $J$ above the plot relate to qualitatively different behaviors of the dissipative soliton.

transient behavior has disappeared, and construct the corresponding bifurcation diagram. Figure 1 shows the result.

\section{BIFURCATION DIAGRAM IN THE REGION OF TRANSITION BETWEEN SOLITONS AND FRONTS}

Figure 1 clearly shows a variety of qualitatively different behaviors, which are labeled with letters from $A$ to $J$ at the top of the diagram. In the following sections, we present a description of each of these different types of solitons.

The pulse profile evolution at $\epsilon=0.782$ is shown in Fig. 2 . The soliton changes its shape periodically in the $t$ direction. This motion is a limit cycle in an infinite-dimensional phase space. This type of solution can be described as a single periodic pulsating soliton. When the value of $\epsilon$ is increased enough, the period of pulsating soliton doubles. This perioddoubling bifurcation occurs at $\epsilon=0.784$. Consequently, in the region around the case labeled $B$ in Fig. 1, the energy, $Q(t)$, oscillates in a way that shows two different values of local maxima, along with two values of local minima. The two minima are very close to each other and, therefore, can

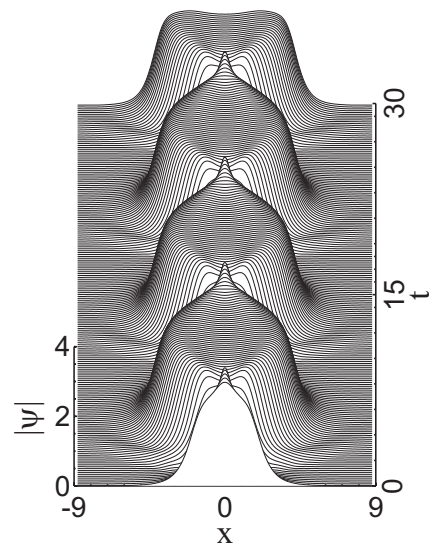

FIG. 2. Propagation profile at $D=1, \delta=-0.1, \beta=0.08, \nu=$ $-0.08, \mu=-0.11$, and $\epsilon=0.782$ (line $A$ in Fig. 1). 

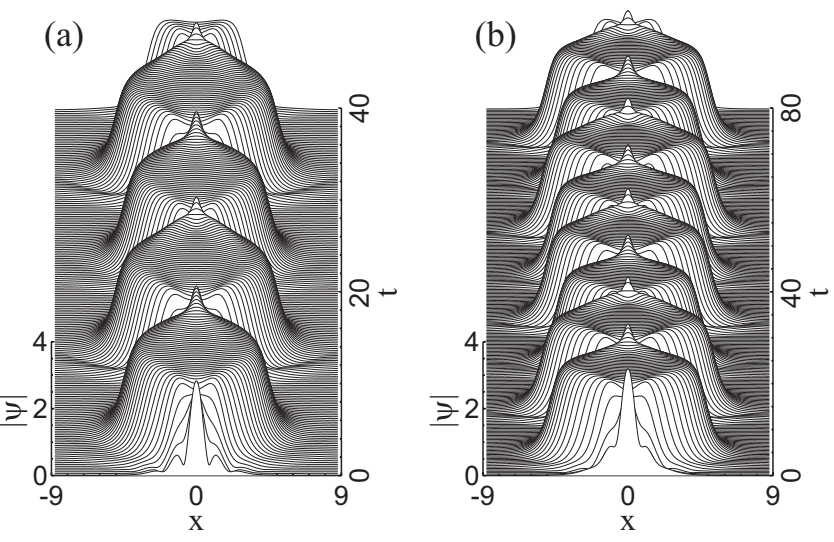

FIG. 3. Soliton dynamics at $D=1, \delta=-0.1, \beta=0.08, \nu=-0.08$, $\mu=-0.11$, (a) $\epsilon=0.793$ (line $B$ in Fig. 1) showing period-doubling. (b) Soliton dynamics at $\epsilon=0.795$ (line $C$ in Fig. 1), showing period-quadrupling.

hardly be distinguished on the scale of the Fig. 1. Branching of the curves corresponding to the maxima are clearly seen in Fig. 1.

A further increase of the $\epsilon$-value, up to the region labeled $C$, shows another period-doubling bifurcation that leads to period-quadrupling behavior with four local minima and four local maxima for $Q$. The evolution of the solution profiles for these cases is shown in Fig. 3. The soliton shape repeats itself every two pulsations in Fig. 3(a), while in Fig. 3(b), there are four pulsations in every closed loop of the limit cycle.

We have calculated the interval $\Delta \epsilon$ for the perioddoubling relative to that for period-quadrupling, namely $(b$ $-0.784) /(0.79615-b)$, where $b=0.794$. This ratio is 4.65 , which is quite close to the generic (period-doubling) Feigenbaum ratio of 4.69 .

Solitons in the region around the line $D$ are unstable. They are pulsating solitons that chaotically change shape as they evolve. The chaotic motion frequently splits the soliton into two parts that are separated and in turn are transformed into chaotic solitons. The train of solitons which originates in this way fills the numerical grid, thus creating, in Fig. 1, the strip with a wide range of values of energy, $Q$. For each soliton, the range of allowed $Q$ values is limited, but the process of their multiplication widens this range to infinity. There are no regular scenarios of soliton evolution in this range of values of $\epsilon$. An example of chaotic evolution of the soliton profile, where there are three solitons in the numerical window, is shown in Fig. 4(a). Each soliton remains localized unless it merges with another one or splits into two new solitons. An example of the latter process is shown in Fig. 4(b). The average distance that each soliton travels before splitting or interacting with another one depends on $\epsilon$ and the density of solitons already existing in the numerical window.

The point $E$ corresponds to a soliton with period-two pulsations with zero velocity, and it is shown in Fig. 5. This dynamic is similar to the one in the region around the point $B$, except for the energy range of $Q$ values, which is now wider-soliton pulsations at point $E$ have a larger amplitude
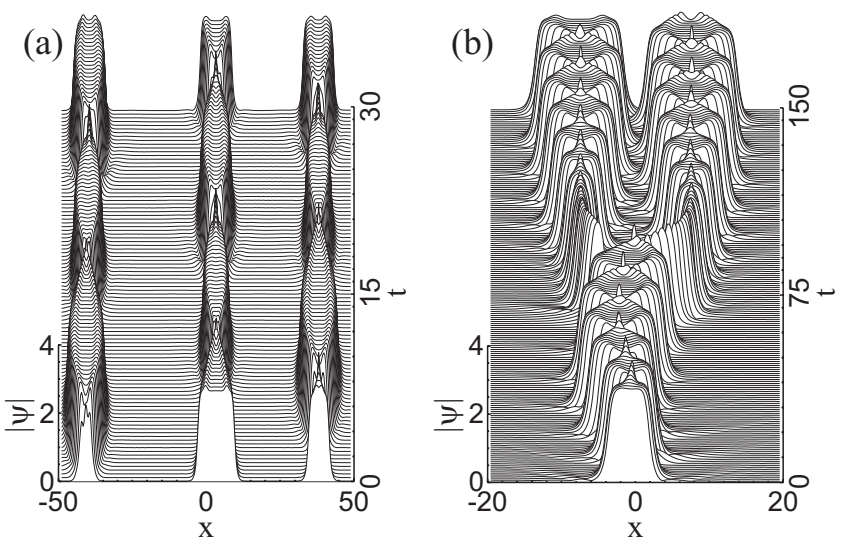

FIG. 4. (a) Typical propagation profile at $D=1, \delta=-0.1, \beta$ $=0.08, \nu=-0.08, \mu=-0.11$, and $\epsilon=0.804$ (line $D$ in Fig. 1). (b) Solitons at this value of $\epsilon$ are unstable and may split into two solitons.

of their oscillations than those at $C$. Increasing $\epsilon$ leads to a bifurcation back to single frequency pulsations. It may happen that the regions $B$ and $E$ can be connected to each other in the complete parameter space. However, investigation of this would require an extended series of numerical simulations.

\section{ZIG-ZAG MOTION OF CREEPING SOLITONS}

Creeping solitons with multiple frequencies of pulsation appear in the region between and around lines $F$ and $G$. There are two wide strips in this region, corresponding to two different creeping solutions which co-exist in a certain interval of values of $\epsilon$, i.e., the lower crescent-like strip in Figs. 1 and 10 and the upper band with higher energy. There are two or more frequencies involved in the motion of these solitons. The combination of two incommensurate frequencies leads to evolution where the energy, $Q(t)$, has local maxima $Q_{\max }$ and minima $Q_{\min }$ in a continuous range of values. Each frequency is related to a particular type of motion, although a complete separation, i.e., the attribution of each frequency to a certain type of motion, seems impossible

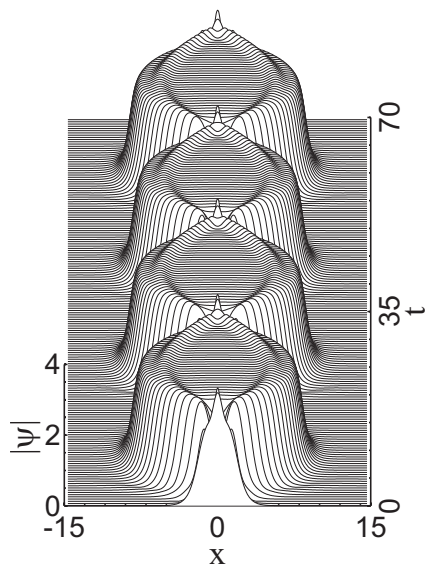

FIG. 5. Propagation profile at $D=1, \delta=-0.1, \quad \beta=0.08, \nu$ $=-0.08, \mu=-0.11$, and $\epsilon=0.821$ (line $E$ in Fig. 1). 

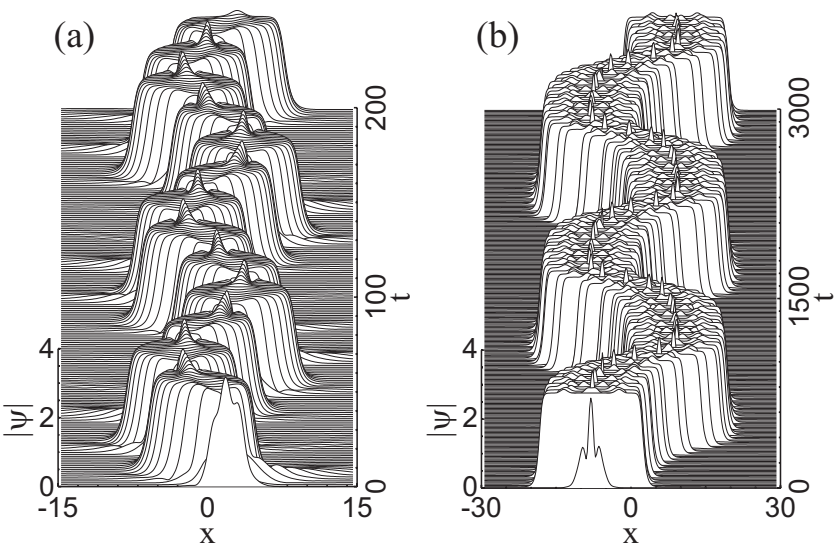

FIG. 6. Propagation profile at $D=1, \delta=-0.1, \beta=0.08, \nu$ $=-0.08, \mu=-0.11$ : (a) $\epsilon=0.828$ (line $F$, lower band in Fig. 1) and (b) $\epsilon=0.835$ (line $G$, upper band in Fig. 1).

in a nonlinear problem. When there are only two frequencies, pulsations occur with the higher frequency (shorter period) whilst the change of the soliton velocity follows the lower frequency (longer period). Two examples of the resulting dynamics in the form of zig-zag motion are shown in Figs. 6(a) and $6(\mathrm{~b})$. The first example is for the lower crescent-like band in Fig. 1 and the second one is for the upper band.

The change in $\epsilon$ from point $F$ to point $G$ results in a change of frequency. For solitons at point $F$ (lower band), the frequency of the velocity change is comparable to the frequency of the soliton width pulsations, while for solitons at point $G$ (upper band), this frequency is much lower than the frequency of the width pulsations. The curves showing energy evolution, $Q(t)$, for these types of motion reveal both frequencies. These curves, for both the $F$ and $G$ cases, are shown in Fig. 7. At point $F$ (lower band), a change in the direction of the motion occurs with a relatively short period compared to the period of the width of the pulsating cycle, whereas at point $G$ (upper band), there are many pulsations inside the "larger" period. In fact, due to the incommensurate frequencies, Fig. 7(a) shows that the soliton energy curve, $Q(t)$, seems to be lacking periodicity, whereas it is actually quasi-periodic. The presence of quasi-periodicity can be studied using a Fourier transform along the $t$ variable. In contrast, the change in soliton energy along the propagation direction at the point $G$ [Fig. 7(b)] clearly shows that there are two frequencies involved in this motion despite the fact that they are incommensurate.

The motion can be more complicated and can involve three frequencies of quite different magnitudes. We have observed the addition of new frequencies for certain $\epsilon$-values in the crescent-like (lower) band of solutions. An example is shown in Fig. 8. In this example, the center-of-mass, defined as $X_{\mathrm{CM}}=\int_{-\infty}^{\infty} x|\psi(x, t)|^{2} d x / Q$, changes with two main frequencies. This is clearly visible in Fig. 8(c). The evolution of the soliton width occurs with the third and highest frequency. This can be seen in the quasi-periodic energy evolution given by the solid line in Figs. 8(a) and 8(b). We show the evolution of the center-of-mass using dashed lines. In Fig. 8(a), it can be seen that $X_{\mathrm{CM}}$ oscillates with a period about five times the period of the oscillations in $Q$. The center-of-mass expe-
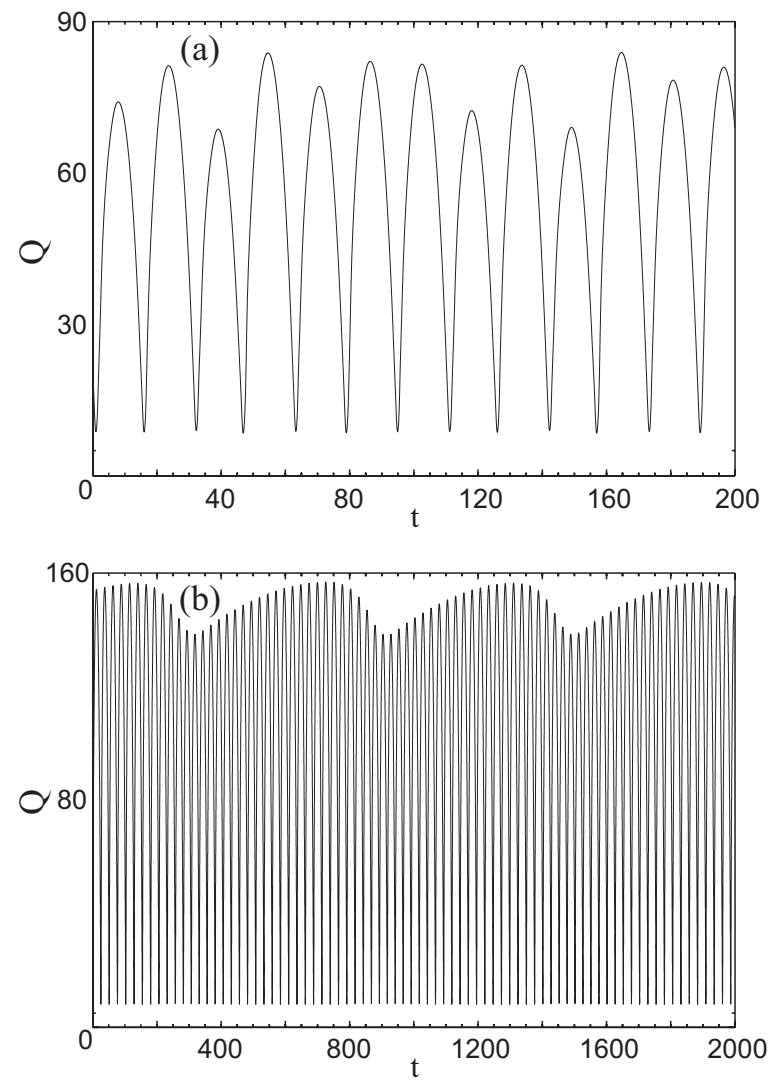

FIG. 7. Soliton energy, $Q$, as function of propagation distance in $t$ direction for $D=1, \delta=-0.1, \beta=0.08, \nu=-0.08$, and $\mu=-0.11$ : (a) $\epsilon=0.828$ (line $F$, lower band in Fig. 1) and (b) $\epsilon=0.835$ (line $G$, upper band in Fig. 1).

riences small abrupt changes every time $Q$ reaches a minimum value. Figure $8(\mathrm{~b})$ shows that the center-of-mass does not follow a strict single periodic motion. Its second main frequency, which is much shorter, is fully apparent in Fig. $8(\mathrm{c})$, where a longer interval of values of $t$ is considered.

The zig-zag motion of the pulsating solitons disappears altogether at the right-hand side end of the crescent-like region of the bifurcation diagram. This motion also disappears at the right-hand side of the upper band. The branch that starts from the upper band corresponds to one-way moving creeping solitons, while the crescent-like lower branch joins the branch of zero-velocity pulsating solitons. These two points of transformation are located between lines $G$ and $H$ in Fig. 1. This zone of the bifurcation diagram is shown at a higher resolution in Fig. 9(a). The branches of pulsating and creeping solitons interact through the small region of bistability shown in Fig. 9(b). The nature of this bistability is explained later in Sec. VIII.

\section{MULTIPLICITY OF SOLUTIONS}

We concentrate on the range of parameters that are in the transition region between pulsating solitons and front solutions. This is relatively narrow in comparison with the whole region of stationary, or even pulsating, solitons. This transition is far from being a trivial phenomenon like a simple 

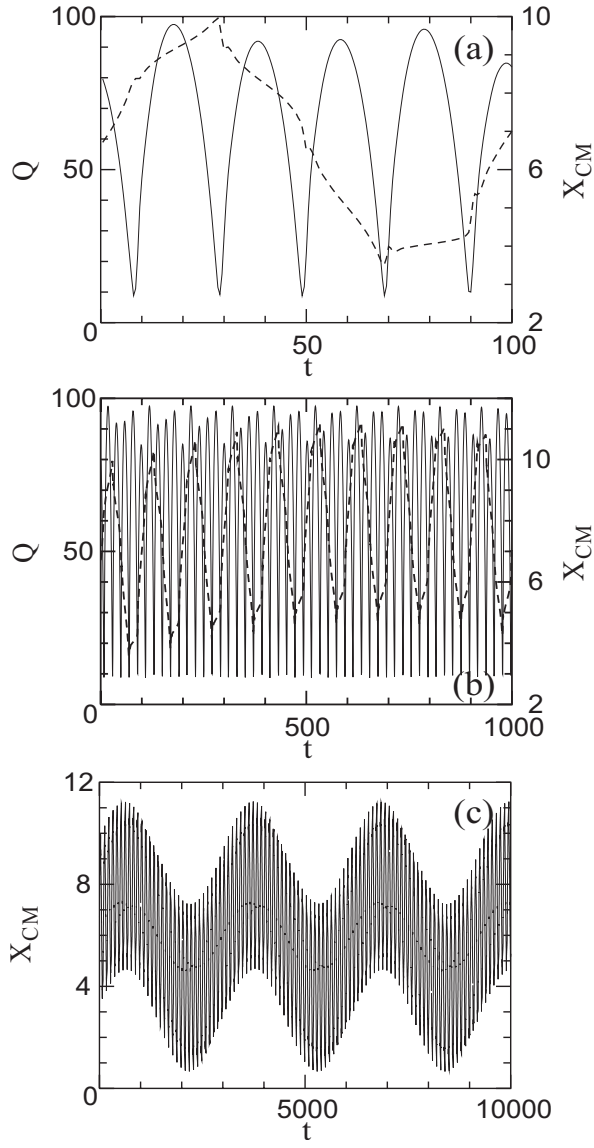

FIG. 8. Soliton energy, $Q$, (solid line) and soliton center-of-mass (dashed line) along propagation direction in $t$ at $D=1, \delta=-0.1, \beta$ $=0.08, \nu=-0.08, \mu=-0.11$, and $\epsilon=0.834$. The same motion is shown on three scales that differ by a factor of 10 from the upper (a) to middle panel (b) and from (b) to (c). The last panel (c) shows only the center-of-mass motion.

increase of the soliton width, as had previously been thought. As a consequence, it is a very dense region with various types of soliton solutions.

Magnifications of the bifurcation diagram reveal a fine structure of transformations between solutions. Figure 10 shows the right-hand side of the bifurcation diagram Fig. 1 with higher resolution. For the sake of clarity, only the maxima of $Q$ for pulsating solitons are presented in Fig. 10, as they show much larger fluctuations than the corresponding minima at the same $\epsilon$. One of the remarkable features of this region is that several solutions may exist for the same value of $\epsilon$. We have found bistability in most of the intervals represented in the figure and tristability in a smaller, but still considerable, interval of values of $\epsilon$.

Each specific part of this diagram can be further resolved using smaller increments of the parameter $\epsilon$ in the simulations. Varying the initial conditions allowed us to find the unstable branches of solitons. In this way, we were able to find all the types of soliton solutions that exist in this region. The parts that contain new types of solutions or bifurcations are enclosed by grey rectangles or circles. They are denoted by the Roman numerals (i)-(iv). These regions are shown in even greater detail in the figures below.
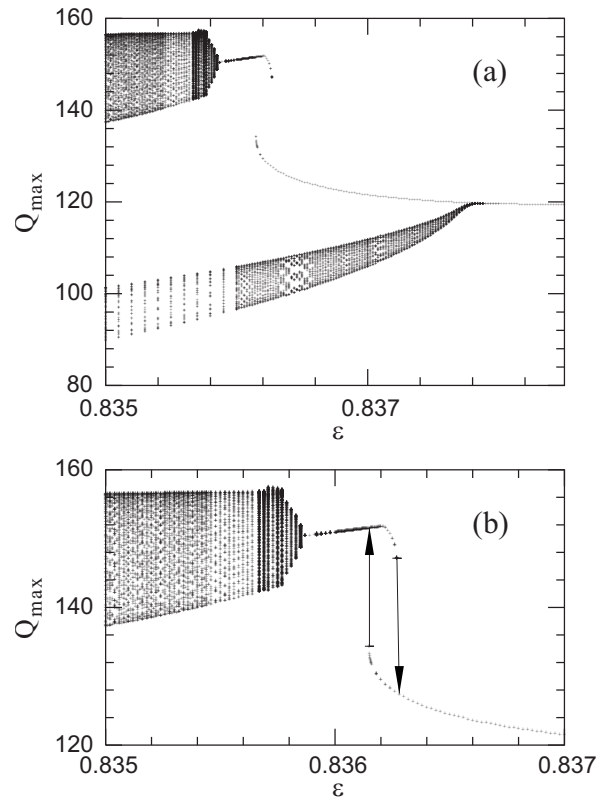

FIG. 9. (a) Magnified region of the bifurcation diagram, shown as (iii) in Fig. 10. This detailed plot shows splitting of the branch of creeping solitons. (b) The branch of solitons above region (iii) that shows bistability which is not resolved on the scale of Fig. 10.

The branch of solutions with the label MP (moving pulse) consists of moving solitons, which were also found in our previous studies [5]. It exists from $\epsilon=0.842$ up to $\epsilon \approx 0.85$. Above this branch, there is another one with higher energy which is denoted CP (composite pulse). This type of solution has a bump in the central part and two fronts located symmetrically on each side of the pulse. The central symmetry of the soliton profile results in it having zero velocity. The CP solution was also known before [5]. The novel feature of this branch that we found in this work is a bifurcation in the middle of its range of existence, i.e., at the point $\epsilon=0.8453$. Here, a composite pulse turns into a pulsating one. To demonstrate this new feature explicitly, we plot the region shown in the rectangle (i) with a higher resolution in Fig. 11. This figure shows the maximum and minimum energies, $Q$, versus $\epsilon$ for just this branch of solitons. For the solution with a stationary profile, the two energies coincide. Thus we have a

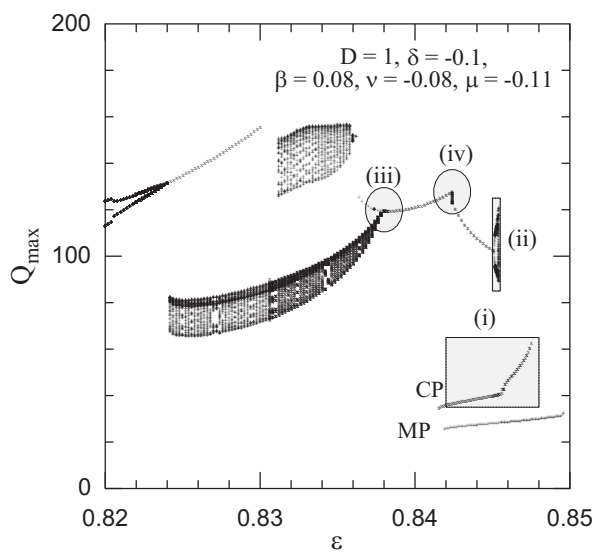

FIG. 10. Detailed bifurcation diagram. 


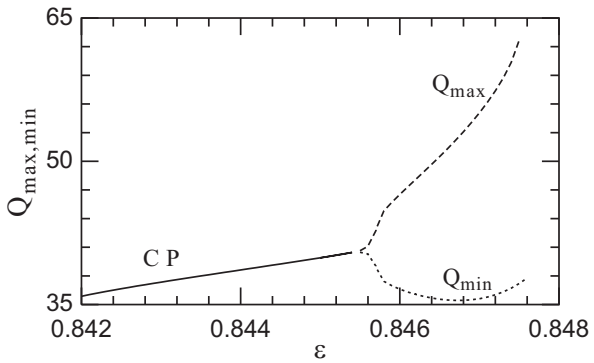

FIG. 11. Transformation of a composite pulse (CP) into a pulsating composite pulse. This corresponds to region (i) of Fig. 10.

single curve up to the point of bifurcation at $\epsilon \approx 0.8453$. Above this point, the energy curve splits, thus demonstrating the pulsating feature of the solution. Hence, this is an example of a Hopf bifurcation-a transition from a fixed point to a limit cycle.

Moving solitons (MPs) exist up to $\epsilon \approx 0.85$. The evolution at point $\mathrm{J}$ of the bifurcation diagram is shown in Fig. 12. The inversion symmetry of the equation relative to the variable $x$ allows for the existence of solitons with positive and negative velocities. They are mirror images of each other. For higher values of $\epsilon$, near the upper boundary of Fig. 10, moving soliton solutions disappear. This is the boundary between solitons and fronts. Localized solutions are transformed into two fronts that move away from each other.

\section{A SEQUENCE OF PERIOD-DOUBLING BIFURCATIONS OF CREEPING SOLITONS}

At least one more type of soliton exists at the same values of $\epsilon$ as MP and CP solutions. The corresponding branch is located above the branches denoted MP and CP in Fig. 10. It starts at the center of the circle (iv) and ends up inside the rectangular box (ii). This is a branch of creeping solitons. An example of a solution for this family is given in Fig. 13. Each side of the branch terminates in a bifurcation.

On the right-hand side of the curve, this branch undergoes a series of period-doubling bifurcations. This can be seen clearly in the magnified part of the diagram enclosed by the

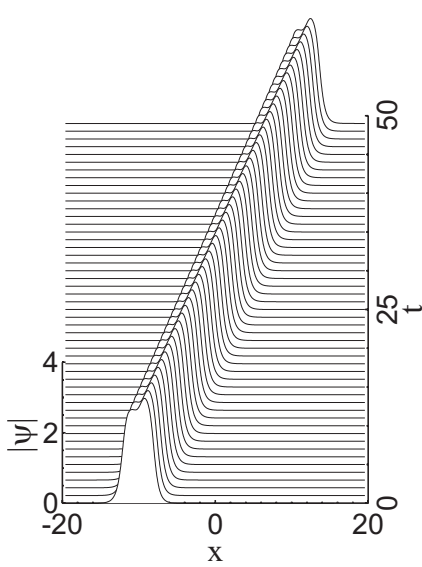

FIG. 12. Propagation profile at $D=1, \delta=-0.1, \beta=0.08, \nu$ $=-0.08, \mu=-0.11$, and $\epsilon=0.846$ (line $J$ in Fig. 1).

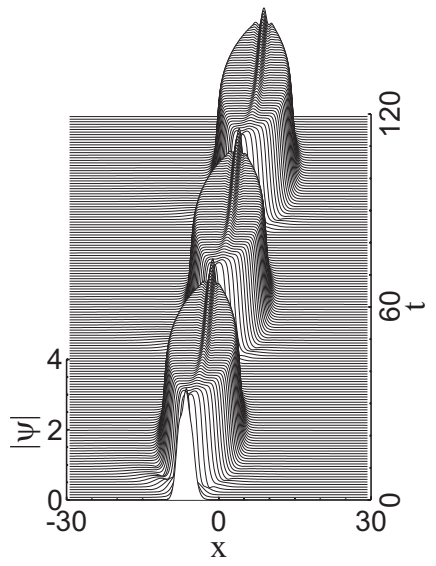

FIG. 13. Propagation profile at $D=1, \delta=-0.1, \quad \beta=0.08, \nu$ $=-0.08, \mu=-0.11$, and $\epsilon=0.844$ (line $I$ in Fig. 1).

rectangle (ii) which is shown in Fig. 10. Our numerical resolution also allows us to observe a period-quadrupling bifurcation at a higher $\epsilon$. The first bifurcation of this series occurs at $\epsilon=0.84511$, while the second one is at $\epsilon=0.84532$. It is very likely that there is the whole sequence of perioddoubling bifurcations on its route to chaotic behavior. In particular, a period-8 solution can also be resolved in the diagram (Fig. 14).

We stress here that this example is the first observation of period-doubling bifurcations of creeping solitons. Earlier, we only reported a sequence of period-doubling bifurcations of zero-velocity pulsating solitons [8]. As a consequence, chaotic behavior of solitons at the end of the route to chaos in the present example is related to moving localized solutions. The velocity of these solitons changes chaotically, always moving the soliton in one direction which is determined by the initial conditions. The branch of chaotic solitons ends at a certain $\epsilon$, with the width of the solitons increasing indefinitely. This evolution leads to a transformation of solitons into two fronts.

\section{SYMMETRY-BREAKING BIFURCATION OF CREEPING SOLITONS}

The point (iv) on the diagram in Fig. 10 is of special interest. The two curves to the left and to the right of this

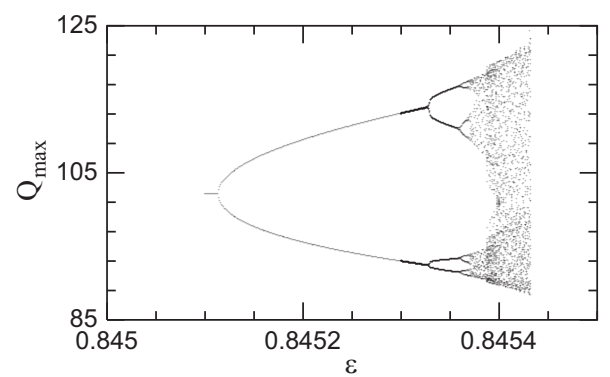

FIG. 14. Period-doubling bifurcations of creeping solitons [region (ii) in Fig. 10]. Bifurcations up to period 8 can clearly be seen. A further increase of $\epsilon$ leads to chaotic dynamics of creeping solitons. 


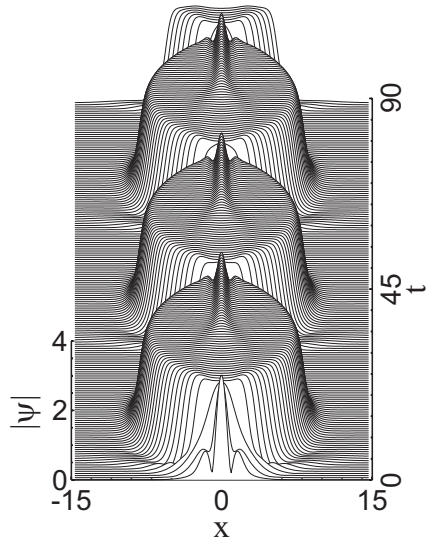

FIG. 15. Propagation profile at $D=1, \delta=-0.1, \beta=0.08, \nu$ $=-0.08, \mu=-0.11$, and $\epsilon=0.839$ (line $\mathrm{H}$ in Fig. 1).

point meet with different slopes, showing that this is indeed the point of another bifurcation. Solitons are pulsating at each side of the point. However, solitons on the left-hand side of the point have zero velocity (Fig. 15), while solitons on the right-hand side have non-zero velocity (Fig. 13).

Our detailed observations show that the curve on the lefthand side of the bifurcation (iv) has a continuation in the form of unstable non-moving pulsating solitons. This continuation is indicated in Fig. 16 by the dashed line. The points on this line have been obtained by using the same propagation technique as for the other branches. However, for the dashed line, the solutions only remained stationary for a limited propagation distance, thus indicating their unstable nature. When we choose symmetric initial conditions, the solutions first converge to zero-velocity pulsating solitons, but these are later transformed into non-zero velocity solutions due to the instability of solitons with zero-velocity. This process of transformation is shown in Fig. 17. The en-

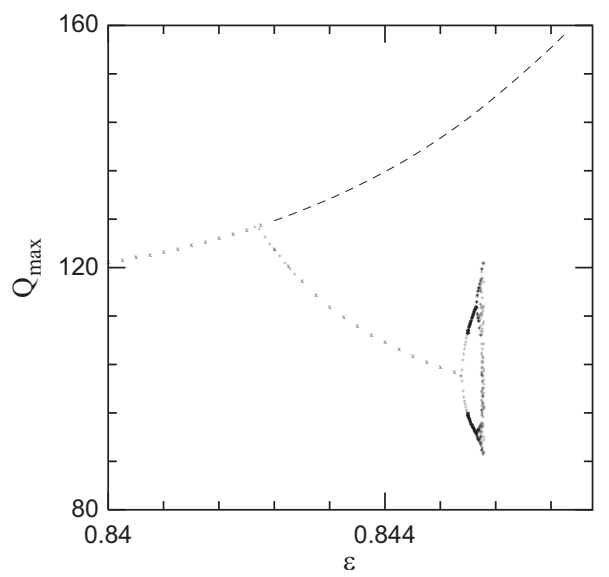

FIG. 16. Symmetry-breaking bifurcation at $\epsilon \approx 0.8425$. The two lines with crosses correspond to stable pulsating solitons. Solitons below the bifurcation have zero velocity. Solitons above the point of bifurcation have non-zero velocity (creeping solitons). This branch is degenerate. Solitons moving to the left have the same energy as the solitons moving to the right. The dashed line corresponds to unstable pulsating solitons with zero velocity. This corresponds to region (iv) of Fig. 10.

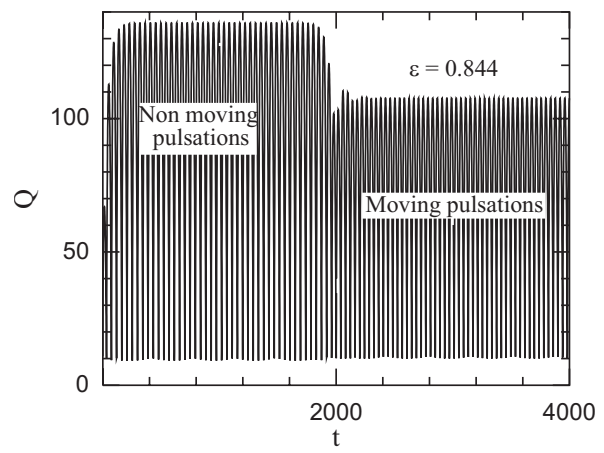

FIG. 17. Energy, $Q$, vs propagation distance, $t$, for $\epsilon=0.844$. Unstable pulsating solitons are transformed into stable creeping solitons.

ergy, $Q$, pulsates for each of the above solutions. However, the amplitude of pulsations is reduced after the transformation from a non-moving pulsating soliton to a creeping one has occurred. Creeping solitons to the right of the point $\epsilon$ $\approx 0.8425$ have a velocity that increases from zero at the point of bifurcation. These observations allow us to conclude that, according to the standard classification in the theory of bifurcations in low dimensional systems [9], the point $\epsilon$ $\approx 0.8425$ is a symmetry-breaking bifurcation. Infinitedimensional systems may have bifurcations with much richer structure than the simple ones appearing in the case of twodimensional phase space. However, the simple classification is valid in higher-dimensional systems as well.

Equation (1) is symmetric with respect to the transformation $x \rightarrow-x$. Localized solutions for this equation can be symmetric, but for this nonlinear system they can also be asymmetric. The transformation $x \rightarrow-x$ converts them into different functions which are also solutions of Eq. (1). In particular, this transformation changes the sign of the soliton velocity. Asymmetric solutions may appear as a result of a bifurcation, as we can see from the present study. An example of a symmetry-breaking bifurcation in the theory of solitons was first found in Ref. [10]. In Hamiltonian systems, this type of bifurcation occurs in an inhomogeneous medium [10]. Dissipative systems allow for symmetry-breaking bifurcations to occur, even in homogeneous media.

The symmetry-breaking instability given in the above example is super-critical. In our simulations, we have obtained a different example of a symmetry-breaking instability, which is sub-critical. The latter creates a small region of parameters where the pulsating and creeping solitons coexist. This example is shown in Fig. 9(b). In this plot, the upper band of solitons, with zig-zag motion, ends with the branch of creeping (asymmetric) solitons that is stable from $\epsilon \approx 0.8358$ up to $\epsilon \approx 0.8362$. The lower branch in this plot corresponds to pulsating solitons (symmetric localized solutions). The two types of solitons co-exist over a small region of $\epsilon$. The transition from one branch to the other one depends on the direction in which the parameter $\epsilon$ is changed. It occurs at the points shown by the vertical arrows. Increasing $\epsilon$ leads to switching from the asymmetric branch to the symmetric one, as shown by the right-hand-side arrow. Decreasing $\epsilon$ results in the transformation shown by the left-handside arrow. The sign of the average velocity of the resulting 
creeping solitons is defined by perturbations in the system. The sub-critical bifurcation leads to a small region with hysteresis.

\section{DISCUSSION}

Dissipative systems with an infinite number of degrees of freedom have rich dynamical behaviors. Leaving aside pattern formation and restricting ourselves to uniquely localized solutions, the number of possibilities still seems to be infinite. On increasing the cubic gain parameter $(\epsilon)$, the localized solutions can pass through regions of no solitons, stationary solitons of various types, pulsating solitons, and then transformation into front solutions. In addition, each boundary contains a multiplicity of bifurcations. Moreover, several solutions can exist simultaneously. Our present study shows that the region of transformation from pulsating solutions to fronts is especially opulent with bifurcations and multistabilities. This occurs even with the simplest modeling when all parameters except $\epsilon$ are fixed. Changing the five other parameters in Eq. (1) as well may result in a multiplicity of other transformations.

The initial incentive to study this problem was our interest in creeping solitons and, in particular, their regions of existence. Clearly, creeping solitons belong to the class of pulsating solitons but they have an additional soliton parameter, viz. non-zero velocity. It is clear then that they can be found near the regions filled with pulsating solutions. As the system is highly complex, we were not able to find some general simple rules governing these transitions. One of the possible techniques that could be used to address these issues is a reduction to a low-dimensional system similar to those performed in Ref. [7]. However, the method of moments would not be able to give an accurate answer because its predictions lack precision. The technique may roughly approximate the boundaries of the regions for creeping solitons, as in the work of Ref. [7], but it cannot describe the whole variety of solutions that we have obtained numerically in the present study. For example, the phenomenon of co-existence of two types of creeping solitons is beyond the reach of any technique which uses any type of reasonably simple trial function. We also doubt that the zig-zag motion of solitons could be described by using a trial function. The fine structure of bifurcations that we found in this work is too detailed to be approximated by any type of reduction to a low-dimensional dynamical system. Individual features of this structure may be modeled, but their relative locations in parameter space are too intricate. Thus, numerical simulations are still the most reliable method for studying the complex dynamics of creeping solitons and dissipative solitons in general.

\section{CONCLUSIONS}

In conclusion, we have studied transitions and bifurcations between different types of pulsating solitons. In particular, when changing the cubic gain parameter, we observed a sequence of period-doubling bifurcations, both for pulsating and for creeping solitons. For the first time, we observed a symmetry-breaking bifurcation for pulsating solitons. We have found zig-zag motion of creeping solitons and the co-existence of two branches of creeping solitons with zig-zag motion. Creeping solitons can also co-exist with other types of dissipative solitons. We have described cases of bistability and tristability.

\section{ACKNOWLEDGMENTS}

This work is supported by the Australian Research Council. The work of J.M.S.C. was supported by the M.E.y C. under contract FIS2006-03376 and by the Australian Research Council.
[1] I. S. Aranson and L. Kramer, Rev. Mod. Phys. 74, 99 (2002).

[2] J. M. Soto-Crespo, N. Akhmediev, and A. Ankiewicz, Phys. Rev. Lett. 85, 2937 (2000).

[3] O. Thual and S. Fauve, J. Phys. (France) 49, 1829 (1988).

[4] S. Fauve and O. Thual, Phys. Rev. Lett. 64, 282 (1990).

[5] N. Akhmediev and A. Ankiewicz, Solitons, nonlinear pulses and beams (Chapman and Hall, London, 1997), Chap. 13.

[6] E. N. Tsoy, A. Ankiewicz, and N. Akhmediev, Phys. Rev. E 73, 036621 (2006).
[7] W. Chang, A. Ankiewicz, and N. Akhmediev, Phys. Lett. A 362, 31 (2007).

[8] N. Akhmediev, J. M. Soto-Crespo, and G. Town, Phys. Rev. E 63, 056602 (2001).

[9] P. Glendenning, Stability, instability and chaos, an introduction to the theory of nonlinear differential equations (Cambridge Univ. Press, Cambridge, 1984).

[10] N. Akhmediev, Sov. Phys. JETP 56, 299 (1982) [Zh. Eksp. Teor. Fiz. 83, 545 (1982)]. 